\title{
AVATARES DE LO «RACIONAL» Y LO «RAZONABLE», CUANDO SON EXIMIDOS DE TENER CARNET DE IDENTIDAD*
}

\section{(como también sobre unos ecos, en Teoría del Derecho, de la perenne dicotomía entre «el científico y el político»)}

\author{
(versión fragmentaria) **
}

\author{
Enrique P. Haba \\ Universidad de Costa Rica
}

RESUMEN. Las teorizaciones «estándar» sobre la argumentación jurídica se basan en unas in-distinciones fundamentales: entre las dos grandes clases de juicios de valor (los categóricos y los instrumentales), entre ciertos meta-lenguajes académicos (Teoría del Derecho) y su lenguaje-objeto práctico (discursos jurídicos efectivos), entre ideal y realidad, entre enunciados descriptivos y prescriptivos, entre verdad y «utilidad», entre conceptuación «amplia» y fórmulas vacías («razonable» y demás), entre recursos discursivos de «política» jurídica y conocimiento propiamente («científico») al respecto, etc. Tales modelos son esencialmente reduccionistas: en cuanto al plano semántico, hacen abstracción de las falacias más corrientes («Síndrome normativista») que presentan los discursos jurídicos profesionales; y en cuanto al nivel pragmático de estos mismos (sus factores psicosociales, sus condicionamientos fácticos en general), lo dejan simplemente fuera de foco.

Palabras clave: argumentación, racional, razonable, juicios de valor, reduccionismo.

ABSTRACT. The Theory of Argumentation -in its "standard» version-is based on some fundamental non-distinctions. This trend of thought does not make clear many capital differences: between the two sorts of value judgments (the categorical ones and the instrumental ones), between a special kind of academic meta-language (Legal Theory) and its practical object-language (real judicial speeches), between ideal and real, between descriptive and prescriptive sentences, between truth and «utility», between concepts with a broad sense and rhetorical empty formulae («reasonable», etc.), between «political» resources of judicial discourses and genuine knowledge (a properly «scientific» one) about legal thinking, and more. The «standard» models bring up an essential reductionist approach: on the semantic level, they look away from the most current fallacies (the "normativist Syndrome") in the lawyers' professional way of reasoning; besides, they simply do not pay attention to its pragmatic level (psycho-social factors, and factual conditionings in general).

Keywords: argumentation, rational, reasonable, value judgments, reductionism.

* Fecha de recepción: 9 de mayo de 2011. Fecha de aceptación: 13 de mayo de 2011.

** Nota de la Redacción: La presentación completa de este estudio excede muy ampliamente los límites máximos establecidos para los artículos que se publican en Doxa. No habiendo pasado inadvertida tal circunstancia al autor, él ha propuesto a la Dirección de la Revista que en esta misma se incluya, de dicho estudio, sólo lo siguiente: sus páginas iniciales, las síntesis ofrecidas en los apartados [0] y II.7, más unas líneas finales. Ello permite anticipar, en la forma más apretada, las principales ideas desarrolladas a lo largo de ese trabajo. La versión completa se publicará en Revista Telemática de Filosofía del Derecho, núm. 14, 2011. 
«... la racionalidad y la irracionalidad. Pocas palabras se usan en manera tan múltiple y contradictoria como estas dos».

MANNHEIM

«... así confío en vencer cualquier obstáculo. A quien no concuerde conmigo, le negaré la facultad de comprender lo racional».

JHERING $^{1}$

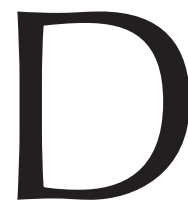

eseo, ante todo, subrayar cuánto me complace que M. ATIENZA haya tomado de manera tan positiva la invitación que le formulé al final de mi contestación anterior. Me llena de satisfacción que, ahora sí, pasa a considerar principalmente IDEAS que son claves para nuestro asunto. Mediante este muy bienvenido giro en sus puntualizaciones se logra, como con razón dice él: «... desplazar la discusión hacia un terreno distinto a aquel en el que estaba planteada... porque éste de ahora me parece más fructífero» (al final del comentario). Inclusive, para no poca fortuna, mía, la paciencia de Manolo hacia mi «prosa suelta y abundante» da para tanto que hasta se manifiesta: «... perfectamente dispuesto a recibir una nueva andanada de críticas de todos los tipos que $\mathrm{H}$. tenga a bien dedicarme» (ibid.). Ante lo amplio que es el espectro de cuestiones de fondo que invita a considerar su nuevo comentario, carezco del heroísmo capaz de permitirme resistir a la tentación de cumplir esa premonición suya.

Las observaciones presentadas en ese segundo comentario no insisten, por lo general, en tomar asiento sobre aquellas mismas suposiciones con respecto a las cuales he demostrado, en mi respuesta anterior, que no tienen sustento alguno en mis propios textos. Así y todo, no podré dejar de hacer ver que, también en esta nueva oportunidad, ATIENZA me adjudica no pocas ideas —otras suposiciones más- que tampoco cuadran con lo señalado en dichos textos, ni en cualesquiera otras publicaciones mías. Lo bueno es que aun esas imputaciones, por más distantes que estén de los puntos de vista que he expuesto en mis trabajos, sin duda conciernen a cuestiones claves del asunto sobre el cual estamos conversando. De tal manera, mis aclaraciones al respecto no significan simplemente una defensa personal frente a ciertas «calumnias», en cuanto a lo teorético, de que ATIENZA me hace objeto (entiéndase esa palabra con toda suavidad, no supongo intenciones aviesas), sino que tales aclaraciones dan, me parece, justamente en el clavo de aspectos fundamentales que los teóricos «estándar» suelen simplemente dejar fuera del ruedo. Por lo demás, es muy cierto que ATIENZA no se limita a «calumniarme», sino que esta vez pone sobre el tapete también unas tesis positivas principales de las que se hace responsable él mismo (en compañía de otros autores), las cuales merecen ser consideradas atentamente. Por ambos lados, me veo ahora en la afortunada circunstancia de complementar mi estudio central con precisiones decisivas sobre cuestiones fundamentales que en este mismo se encuentran apenas insinuadas o no fueron tocadas del todo.

${ }^{1}$ K. MAnNheIm, El hombre y la sociedad en la época de crisis, Buenos Aires, 1969, 38. R. VON JHERING, Broma y veras en la Jurisprudencia, Buenos Aires, 1974, 349. 
En efecto, la nueva intervención de ATIENZA lleva a poner sobre el tapete puntoseje como los siguientes: tipos de juicios de valor y su papel en las decisiones judiciales - diferencia esencial entre los planos de lenguaje considerados-, tipos de pruebas requeridas para sustentar las tesis de Teoría del Derecho - precisiones fundamentales con respecto al funcionamiento discursivo de términos como «racional» $\mathrm{y}$ «razonable» (¿`se comportan, o no, a modo de «fórmulas vacías»?)—, qué papel pueda caberle a unos estudios de Teoría del Derecho para «orientar»/《guiar» al jurista en sus razonamientos y así «ayudar» a «mejorar» las fundamentaciones de los jueces —aquilatar si tienen o no «sentido» los razonamientos jurídicos habituales y saber si es posible propiamente «justificar» las decisiones judiciales-, ¿inciden habitualmente «argumentos falaces» en el «centro» de las discusiones jurídicas o antes bien estas últimas son cuestión de «discrepancias» libres de aquéllos? — ¿no vale mucho la pena, por lo general, «diferenciar con precisión los elementos descriptivos y prescriptivos» en los razonamientos jurídicos? - cognitivismo axiológico normativista (¿en vez de «positivismo lógico»?) como esencial artículo de fe para las teorizaciones «estándar» (por cierto, considero muchísimo más «fructífero» discutir sobre tales cuestiones, que continuar multiplicando al infinito la producción de divertimentos como, p. ej., el cúmulo de traídas y llevadas fijaciones académicas al gusto para la etiqueta «positivismo» jurídico o el listado de los dibujitos lógico-deónticos).

He ahí las principales cuestiones de que tratará el presente examen. Luego de ofrecer una visión sinóptica [0], apenas aforística, de las principales ideas que desarrollará este texto, haré constar algunas aclaraciones preliminares de orden general [I]. Luego pasaré a «desmenuzar» el quid de los principales aspectos de fondo suscitados por mi comentarista. La sección principal [II] presenta elucidaciones que, si bien arrancan de puntos de vista planteados en ese comentario suyo, conciernen directamente no sólo a Teoría de la Argumentación, ni sólo a «reproches» específicos que me hace él, sino que ellas llaman la atención sobre unas cuestiones básicas para los estudios de Teoría del Derecho en general. Las secciones que siguen se ocupan respectivamente de: [III] aclarar ciertos nuevos malentendidos capitales de ATIENZA sobre las aproximaciones realistas al estudio de los razonamientos jurídicos; [IV] añadir aún, finalmente, algunas observaciones complementarias sobre las limitaciones esenciales (tratamiento reduccionista) que comporta el tipo «estándar» de aproximación a dichos razonamientos.

\section{SUMARIO (muy sintético):}

0. Ideas cardinales.

I. Puntualizaciones liminares: 1. Estilos contrapuestos. 2. ¿A qué se le ha de llamar teoría «estándar»? 3. ¿Una «conspiración» del silencio...? 4. ¿«Positivismo lógico»?

II. Precisiones metodológicas fundamentales: 1. Tipos de juicios de valor, planos de lenguaje. 2. El asunto de las «pruebas» (¿qué métodos de investigación?, ¿qué tribunales?). 3. ¿«Concepción estrecha» o una mucho «más amplia» acerca de «racional», «razonable», etc.? (papel de tales «fórmulas vacías»). 4. Contra el capital reduccionismo que comportan las visiones racio-argumentativistas. 5. ¿Es posible, y cómo, «ayudar»a «mejorar» («El político y el científico»)? 6. Eventual «fertilidad» cognoscitivo-heurística en Teoría del Derecho. 7. Recapitulación.

III. Precisiones sobre «realismo» jurídico: 1. ¿Tienen «sentido» los razonamientos jurídicos? 2. ¿Las tesis realistas implican negar que las sentencias se pueden «justificar»? 3. ¿Las aproximaciones realistas «no pueden» servir para «orientar»/«guiar» al jurista práctico? 
IV. Precisiones complementarias sobre reduccionismo de las teorizaciones «estándar»: 1. Cognitivismo axiológico normativista («mons razonabilis»). 2. La «cirugía estética» razonabilista. 3. ¿Es falso que la teoría «estándar» se «desentiende» del Síndrome normativista? 4. ¿Las discusiones jurídicas se asientan en «argumentos falaces» o consisten esencialmente en unas «discrepancias» sin más? 5. ¿No vale la pena «diferenciar con precisión los elementos descriptivos y prescriptivos»?

\section{IDEAS CARDINALES}

Ese comentario de ATIENZA contiene dos rubros de elucidaciones: una cara «negativa» y una «positiva», por así decir. Tienen finalidad «negativa» sus observaciones críticas sobre varias ideas fundamentales que él me imputa. Como «positiva», en cambio, bien puede calificarse su contraposición, frente a aquello «negativo», de ciertas ideas a las que reconoce validez primordial él mismo, las cuales coinciden con postulados básicos compartidos por los autores «estándar» en general. Tanto lo primero como lo segundo se asientan en determinadas pre-suposiciones, respectivamente, de las cuales depende —al menos en buena parte- el posible acierto de lo afirmado por ATIENZA en función de ellas. Pero si estas mismas resultan dudosas, por decir lo menos, con ellas tambalean igualmente, o hasta se vienen abajo del todo, las principales aseveraciones negativas o positivas en que mayor hincapié hace ATIENZA.

- ÁNGULO NEGATIVO. La presuposición esencial de las críticas formuladas por ATIENZA es que mis puntos de vista fundamentales son los mismos que, o en todo caso muy parecidos a, los del positivismo lógico (así lo señala expresamente) (infra, I.4). Según mi comentarista, yo aplicaría justamente esos cartabones para enjuiciar el pensamiento jurídico. Ahora bien, lo decisivo en este caso no es simplemente que ATIENZA crea tal cosa, no poco alejada de la verdad. Mas tal creencia empapa hasta tal punto sus observaciones específicas a mi respecto, que le lleva a considerar antes bien lo que al respecto pudiera tal vez haber dicho algún positivista lógico (ignoro si hay o haya habido tal específicamente), en lugar de concentrar sus objeciones sobre lo escrito en mis propios textos. Así es cómo esa capa de «positivismo lógico» con que ATIENZA cubre, por su propia cuenta, lo señalado en esos textos mismos, logra que su «lectura» de éstos venga a «decirle», particularmente a él, ni más ni menos que dos órdenes de cosas: por un lado, le hace suponer que sostengo ideas que en modo alguno sostengo (en mis trabajos no se afirma nada por el estilo, ni explícita ni implícitamente); por el otro, hace que en tal lectura no se puedan echar de ver, en cambio, mis ideas principales que no calzan con dicha presuposición. Una y otra vez toparemos con esas dos clases de malentendidos; no sería extraño que, en efecto, tengan su origen en dicha presuposición generalizada.

$\square$ ÁNGULO POSITIVO. La otra cara de las consideraciones efectuadas por ATIENZA son las tesis avaladas por él mismo, especialmente unas que subraya en esta segunda ocasión. Ello tiene su propia base epistemológica, la cual depende sobre todo de cierto «artículo de fe»: al parecer, ATIENZA se basa en un cognitivismo axiológico, la suposición de que hasta los fines/valores últimos es dable «conocerlos» mediante tales o cuales criterios propiamente «objetivos». En este caso, se trata de un cognitivismo que presenta la singularidad de ser específicamente normativista-«razonabilista» («mons razonabilis») (infra, IV.1). Ahora bien, por ser ello un postulado de nivel último en la 
concepción que ATIENZA defiende, vale decir, un principio fundamental puesto allí como axiomático (funciona como artículo de fe básico en ella), no es posible pedir ningún tipo de «pruebas» al respecto. Los axiomas no se demuestran, pues son ellos mismos quienes constituyen el punto básico de arranque, «ratio» última, para las demostraciones posibles dentro del sistema de pensamientos — en este caso es el «razonabilismo» argumentativista - asentado sobre aquéllos. Por eso, mientras que acerca de unas afirmaciones «negativas» de ATIENZA podré demostrar que no corresponden a los hechos por él invocados (en este caso, hechos discursivos: mis propios textos), en cambio con respecto a esas bases «positivas», al tratarse de postulaciones que se toman ahí como válidas en sí y por sí mismas (axiomas), no es posible ni demostrarlas ni falsarlas: cada quien sabrá si «cree» en ellas o no. Lo que resulta posible, sí, es hacer ver ciertas derivaciones lógicas de esos axiomas y también unos efectos empíricos resultantes de aplicarlos. De tal manera, quien sostiene los axiomas se hace solidario también con esos dos órdenes de consecuencias vinculados directamente con aquéllos, las cuales no pocas veces son disimuladas por la doctrina en cuestión. Este último es el caso, según veremos, de las teorizaciones argumentativo-razonabilistas.

Por ambas vías, la «negativa» y la «positiva», ATIENZA pone sobre la mesa una serie de afirmaciones que vienen a conjugarse, todas ellas, hacia el mismo punto fundamental: desviar la vista del reduccionismo propio de las teorizaciones «estándar». La idea central del presente trabajo es salir al paso de tales intentos de justificación para ese modo tan unilateral de abordar las cuestiones jurídicas. De tal manera procedo a complementar los señalamientos que en mi estudio básico efectué sobre la naturaleza esencialmente «escapista» de dicha orientación; las puntualizaciones que presentaré aquí traen a colación precisiones que son de importancia primordial, a mi juicio, en cuanto a aspectos que no fueron especificados suficientemente en dicho estudio.

$$
* * *
$$

Como el presente escrito es un tanto detallado, voy a adelantar ya, sólo aforísticamente, mis afirmaciones principales (indicando también dónde se encuentran desarrolladas en el presente trabajo). Así el lector queda en condiciones de elegir a cuáles de ellas desea prestarle su atención, si prefiere no detenerse en la totalidad de esos aspectos que considero oportuno aclarar.

- NO he sostenido, ni directa ni indirectamente, ninguna de las principales ideas siguientes (ATIENZA me las imputa):

- Que la base metodológica de los estudios de Teoría del Derecho sería deseable conformarla a las tesis principales del «positivismo lógico» $[\mathrm{I} .4]^{2}$.

- Que los juicios de valor no son susceptibles de someterlos a discusiones «racionales» [II.1, III.1-2].

- Que los razonamientos jurídicos no tienen «sentido» [III.1].

- Que las decisiones judiciales no puedan ser «justificadas» mediante fundamentaciones «razonables» y hasta unas «racionales» —es decir, con base en ciertos modelos normativos de razonamiento, que en mayor o menor medida son ideales-; no

${ }^{2}$ Las cifras colocadas entre corchetes remiten al numeral donde hay unas explicaciones sobre ese punto en el presente trabajo. 
impugno que tales argumentaciones constituyan la situación más general, ni que estas mismas tengan cierto «peso» —unas veces más, otras menos- entre los factores reales que co-determinan la parte resolutiva de las sentencias [III.2].

- Que las falacias no son «contextuales» [IV.4.i].

- Niego, asimismo, que de mis propias consideraciones se desprenda nada parecido a lo siguiente (ATIENZA lo «deduce», por su propia cuenta, de lo que él supone son ideas mías al respecto):

- Que las puntualizaciones realistas acerca de los discursos judiciales (en todo caso, las mías) «no pueden» servir para «orientar»/《guiar» al jurista práctico en sus tomas de decisión (sentencias y demás), o que acaso los realistas no quieran «ayudar» a «mejorar» los discursos jurídicos [III.3].

- SOSTENGO, sí, las siguientes tesis metodológicas fundamentales:

- Que también a los estudios de Teoría del Derecho se aplica la dicotomía señalada por WEBER en cuanto a orientarse como «político» o como «científico» [II.5].

- Que una cosa es la posible verdad de tales o cuales tesis sostenidas en el seno de dicha Teoría, mientras otra cosa es la eventual utilidad que ellas mismas puedan tener de hecho en las propias prácticas de los juristas profesionales [II.5].

- Que por lo general esas tesis no alcanzan a tener, en el mejor de los casos, más que alguna «fertilidad» cognoscitivo-heurística («ideas para tener en cuenta», entre las cuales puede haber también unas que se invoquen a título de parangón como modelos normativos de razonamiento) [II.6].

- Que es fundamental saber si un juicio de valor se entiende como «categórico» o como «instrumental», y que la abrumadora mayoría de los juicios de valor utilizados por los juristas corresponden decisivamente (al menos en parte) al segundo tipo [II.1.i, II.4].

- Que también es capital no confundir entre los alcances que pueda tener un meta-lenguaje como tal —así la Teoría del Derecho—y los alcances que tienen los discursos mismos del lenguaje-objeto considerado —así los razonamientos de los propios juristas prácticos- [II1.ii].

- Que palabras como «racional», «razonable», etc., se suelen usar como fórmulas vacías (o, en todo caso, semivacías) — digamos: sin «carnet de identidad»— en los discursos jurídicos efectivos [II.3].

- No quiere decir que esos términos no se pueden definir teoréticamente en formas que no sean ya tan manipulables, sobre todo «racionalidad» (cfr. M. WEBER) - pero no es así cómo aparecen en dichos discursos mismos- [II.3].

- Que el carácter de generales que tienen las tesis sostenidas en Teoría del Derecho conlleva, debido a la naturaleza misma de esta materia, que si bien es posible señalar ciertos indicios de «prueba» con respecto a ellas y hacerlas objeto de ciertos «controles» discursivos, empero no resulte posible someterlas a «métodos rigurosos» de verificación empírica [II.2].

- Que los «argumentos falaces» (Síndrome normativista y demás) suelen incidir importantemente en el «centro» de las discrepancias entre los juristas prácticos [IV.4].

- Que para alcanzar «transparencia» en las fundamentaciones jurídicas es de la más alta importancia saber distinguir netamente, en el seno de cada una de ellas, en- 
tre elementos «descriptivos» y elementos «prescriptivos», porque estos dos tipos de elementos corresponden respectivamente a «gramáticas» cuyos requisitos de «prueba» son de muy distinta naturaleza «categorial» para los primeros que para los segundos [IV.5].

- Que, sin perjuicio de lo anterior, desde luego ambos tipos de enunciados se pueden «combinar» entre sí, como incluso: $a$ ) es corriente que la selección de los elementos descriptivos venga determinada por unos elementos de juicio valorativo-prescriptivos, y b) que el alcance jurídico otorgado a los segundos dependa de comprobaciones acerca de los primeros [IV.5].

- Que nada de lo señalado significa descartar la presentación de modelos ideales para el razonamiento jurídico (hasta yo mismo he ofrecido uno de «cuatro pasos»), ni negar que eventualmente algunos de éstos puedan tener cierto valor heurístico para juristas que se resuelvan a tomarlos en cuenta; siempre y cuando no deje de hacerse la advertencia, pero en forma inconfundible, de que tales modelos no constituyen comprobaciones sobre cómo el Derecho funciona realmente (aunque de seguro ellos comprendan también unas similitudes con razonamientos jurídicos habituales) [IV.5].

- Que el cognitivismo axiológico normativista en que se basan las teorizaciones «estándar» no es otra cosa que un postulado de fe (... del «wishful thinking» al «mons razonabilis») [IV.1].

- Que la diferencia esencial entre las aproximaciones «estándar» y los enfoques realistas reside en que las primeras constituyen unas visiones muy reduccionistas acerca de los discursos jurídicos; por lo demás, las maneras cómo dichas aproximaciones presentan a estos últimos constituyen una «cirugía estética» al respecto [II.4, IV].

- Que las aproximaciones realistas, para ser tales, no tienen por qué desconocer la importancia práctica efectiva que puedan tener las argumentaciones presentadas en los tribunales (por ende, tampoco descartarán la pertinencia de tales o cuales observaciones contenidas en teorías «estándar», en cuanto éstas no confundan unos ideales con dichas prácticas en sí mismas) [II.6].

- Que todo cuanto de dichas teorías sea rescatable para orientar realmente unas prácticas jurídicas, o al menos lo sea como beurística para mejor conocimiento de ciertos aspectos de estas mismas, ciertamente será bienvenido por el realista (p. ej., ello puede caber dentro del 1 .r $^{\text {er }}$ «momento» del modelo procedimental jurídico-realista de «cuatro pasos» expuesto por mí) [II.6].

- Pero el realismo jurídico ${ }^{3}$ no se queda ahí (conformarse con elucidaciones limitadas a poner sobre la mesa unos topoi del pensamiento jurídico, sean o no «razo-

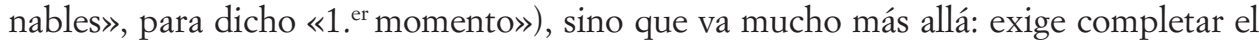
examen meramente semántico-argumentativista (aquel «1 ${ }^{\mathrm{er}}$ momento») con decisivas consideraciones de orden discursivo-pragmático (así, al añadir los «tres pasos» de análisis indispensables para esclarecer lo más posible las dimensiones prácticas de los argumentos presentados en su «paso» inicial) [II.6].

$$
* * *
$$

3 Cuando escribo «el» realismo o «el» realista, no es cuestión de entender que ignoro diferencias y también contradicciones entre distintos autores conocidos como «realistas». 


\section{II.7. Recapitulación (muy aforística) (de la sección II)}

1. Confusiones Categoriales. Los planteamientos de Atienza incurren en unos básicos «errores categoriales», consistentes en no distinguir entre planos de pensamiento que, si bien están relacionados, no son necesariamente homogéneos entre sí, aunque sus respectivos contenidos pueden presentarse siendo llamados de manera igual o muy similar. ATIENZA «mete en un mismo saco»: por un lado, el plano de los juicios de valor categóricos y el plano de los juicios de valor instrumentales; por el otro, el plano de los razonamientos jurídicos profesionales corrientes en sí mismo (lenguajeobjeto) y el plano de las elucidaciones de Teoría del Derecho (meta-lingüísticas) acerca de los primeros. Tales indistinciones originan múltiples confusiones, tanto para saber a qué exactamente entienda referirse ATIENZA en muchos de sus pasajes (¿¿a cuál de los dos planos de un nivel o si es a ambos a la vez?) y también porque las respectivas exigencias de fundamentación son disímiles.

2. «PRUEBAS». No queda para nada claro qué clases de «pruebas» —si es que hacen falta- pide ATIENZA para los enunciados de Teoría del Derecho, en general. Pareciera que solicita unas de cierto tipo, «métodos muy rigurosos», para tesis que no le simpatizan, mas no ofrece pista alguna acerca de cuáles serían las requeridas ( $¿ \ll m e-$ tódicas» o no?, ¿‘sometidas a qué «rigores» o libres de ellos?) para solventar tesis como las sostenidas por él mismo y en general las de los teóricos «estándar»; a no ser que, para él, sólo las primeras requieran comprobación, mientras que las segundas estarían, por principio, eximidas de toda exigencia al respecto.

3. LO «RACIONAL», LO «RAZONABLE», ETC. Para términos claves como «racional» y «razonable»—también: «universalidad», «coherencia». etc.—, según ATIENZA es inadecuado hacerlos objeto de una «concepción estrecha» (así la de WEBER); considera muy preferible que ella sea mucho «más amplia». La cuestión, no respondida por él, es: ¿«más amplia» en qué y hasta dónde? (aun si nos abstenemos de pregunta el «para qué»). ATIENZA no suministra ninguna indicación acerca de cuál sería por lo menos algún «poco» de contenido descriptivo, «algo» que permita diferenciar intersubjetivamente tal mínimo - ; aunque fuere solo esto! - frente a cualquier otra suerte de razonamientos. Tampoco ofrece ninguna pista acerca de cómo saber (intersubjetivamente) si lo «razonable» es A o no-A, cuando esto se halla en disputa; o si será que basta con que determinados locutores digan que es así, ya sea lo uno o lo otro, para tenerlo por tal. El uso de esos términos suele responder, de hecho, al encanto irresistible de conjurarlos como fórmulas vacías. ATIENZA dice que hay, a pesar de todo, un uso «legítimo» para tales palabras, mas no señala cómo distinguir (intersubjetivamente) este mismo frente a los eventuales usos «ilegítimos».

4. REDUCCIONISMO. La visión «estándar» acerca de los discursos jurídicos es esencialmente reduccionista y en general extra-pragmática. Opera como una autocensura, de quien se ciñe a ella, limitándole a fijarse en ciertos aspectos abstracto-semánticos de dichos discursos (incluso dejando fuera de foco las falacias más corrientes en dichos discursos) y haciendo en general abstracción de las dimensiones pragmáticas de ese lenguaje (sobre todo, de aquellas que resultan ser las más decisivas de hecho).

5. «MEJORAR». En la forma en que lo plantea ATIENZA, la cuestión de cómo «ayudar» a «mejorar» los discursos jurídicos de los jueces no se puede examinar propiamente, ni siquiera con cierto grado de aproximación, pues se omiten las aclaraciones 
más elementales para determinar qué sea específicamente lo que se desea «mejorar»y cuáles serían los parámetros para juzgar al respecto. Por lo demás, él no distingue entre verdad y «utilidad» en cuanto a los contenidos teoréticos de los eventuales proyectos de «mejora». Al parecer, da simplemente por presupuesto el principio platónico de que ambos planos se corresponden por fuerza: Verdad = Bien (útil). De tal modo pasa por encima, sin más, de la conocida antinomia entre «El político y el científico» (WEBER); o sea, desconoce la neta diferencia categorial (advertida por KELSEN) que hay entre a) «ciencia (conocimiento) jurídica», y b) la «función jurídico-política» en sí misma [utilidad] de los discursos desempeñados en la práctica profesional de los juristas. Al fin de cuentas, el reduccionismo propio de las teorías «estándar» obedece justamente a que, para poder ser «útiles» (política) — isi acaso!—, ellas suscriben sin más el sacrificium intellectus de «no mentar la soga (Síndrome normativista y demás) en la casa del ahorcado»...: se conforman con «decir lo justo» (no-callar-pero-callar), así le llama ATIENZA.

6. POSIBLE «FERTILIDAD». Abstracción hecha de la medida en que la Teoría del Derecho procure ser «política» (en el sentido señalado: 5), es verdad que algunos de los saberes producidos en esa disciplina pueden tener cierta «fertilidad» cognoscitivoheurística («ciencia» acerca del Derecho), en mayor o menor medida. Inclusive las teorizaciones «estándar» contienen buenas porciones de ello, aun dentro del reduccionismo que las caracteriza. Eso sí, los contenidos auténticamente cognoscitivos están en condiciones de llegar a ser mucho más amplios, y sobre todo ir muchísimo más a fondo, si el investigador se inspira, a diferencia de aquellas teorizaciones, en el principio heurístico de no-callar. Justamente este último constituye el principal objetivo de la corriente realista en general. Es así cómo, en efecto, buena cantidad de los trabajos que pueden considerarse como pertenecientes a ella han sacado a luz aspectos decisivos de la discursividad jurídica, justamente unos elementos de juicio que en los otros tipos de estudios de dicha Teoría — los ampliamente dominantes en su seno (así en la generalidad de los pertenecientes a teóricos «estándar») — se quedan simple y llanamente «entre bambalinas».

No podría finalizar mi comentario sin poner especial énfasis en subrayar aquello que, justamente por haber sido llevada adelante nuestra discusión de la manera en que ha tenido lugar, debiera llamar la atención por encima de cualquier otra cosa en la presente controversia. Tengo para mí que su resultado más estimulante es hacer ver que aun en nuestros tiempos, a pesar de todo el encerramiento académico en capillas cuyos respectivos dogmas cada una exige tener por intocables, sigue siendo todavía posible llevar a cabo un debate de ideas SIN condicionarlo a no impugnar esos dogmas mismos. Pues sí, se trata ni más ni menos que del viejo principio cardinal proveniente de la Ilustración:

«... la ausencia de restricciones al derecho de discusión y de crítica, aplicado no solamente a los resultados parciales, sino a los FUNDAMENTOS y a los MÉTODOS» ${ }^{4}$.

Se dirá: ¿quién va a estar en contra de un principio como ése? De palabra, ipor supuesto que nadie! (en el medio académico). Mas en los hechos... La circunstancia de

\footnotetext{
${ }^{4}$ R. ARON, «Introducción», en M. WebER, El político y el científico, Madrid, 1994, 28 (vers. e.p.h.).
} 
que un escritor bien conocido de nuestra disciplina, y por añadidura director de la más connotada revista de esa materia en idioma español, haya abierto espontáneamente las páginas de ella misma a un debate de tal naturaleza, vale decir, sabiendo de antemano que su contendor no se iba a andar allí con «paños tibios», no es cosa de hallar así como así en nuestros círculos profesorales. Por eso, ¿qué importa, al fin de cuentas, si en definitiva no serán muchos, posiblemente, quienes terminen estando de acuerdo con razones como las presentadas por mí en este debate? Yo sería el más feliz de los mortales si el ejemplo suministrado por mi paciente crítico consigue convencer, ;sobre todo!, de proceder como M. ATIENZA en lo más importante: que también otros acepten poner la justificación de sus propias posiciones «en juego» ante comentaristas muy poco complacientes. Mentiría si me manifiesto como optimista al respecto, pero también es verdad que a veces hasta los buenos ejemplos logran provocar algún que otro eco análogo por ahí. 